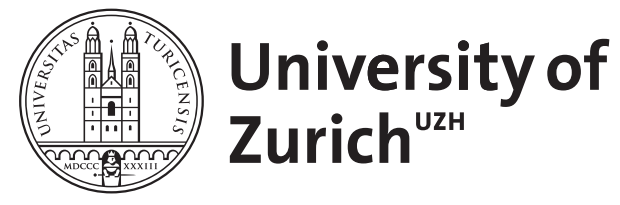

Zurich Open Repository and Archive

University of Zurich

University Library

Strickhofstrasse 39

CH-8057 Zurich

www.zora.uzh.ch

Year: 2010

Laboratory diagnostics in acute poisoning : critical overview

Rentsch, K M

DOI: https://doi.org/10.1515/CCLM.2010.295

Posted at the Zurich Open Repository and Archive, University of Zurich

ZORA URL: https://doi.org/10.5167/uzh-45933

Journal Article

Published Version

Originally published at:

Rentsch, K M (2010). Laboratory diagnostics in acute poisoning : critical overview. Clinical Chemistry and Laboratory Medicine, 48(10):1381-1387.

DOI: https://doi.org/10.1515/CCLM.2010.295 
Review

\section{Laboratory diagnostics in acute poisoning: critical overview}

\author{
Katharina M. Rentsch* \\ Institute for Clinical Chemistry, University Hospital \\ Zurich, Switzerland
}

\begin{abstract}
Laboratory diagnostics play an important role in the treatment of patients with acute poisoning. The classical clinical chemistry and hematology tests help initiate supportive treatment, and specialized methods enable elucidation of the poisons involved. In this context, two different analytical approaches are used: the direct quantification of a potentially involved compound or screening procedures looking either for a distinct drug class or a wide variety of different compounds. The most common tests are immunoassays, which have the advantage of being fast and highly automated. These assays are available for the substances which are often involved in intoxications. The other analytical technique which is widely used is hyphenated chromatography consisting of either high-performance liquid chromatography or gas chromatography as chromatographic systems and detection with a diode-array or mass spectrometer. Whereas gas chromatography mass spectrometry screening procedures have been known for a long time, liquid chromatography mass spectrometry screening methods are now developed by different research groups and still need to prove their reliability. In this review, the different analytical technologies and their application will be discussed.

Clin Chem Lab Med 2010;48:1381-7.
\end{abstract}

Keywords: chromatography; immunoassay; mass spectrometry; poisoning.

\section{Introduction}

Depending on the severity of the clinical symptoms and the substances involved, intoxications may be very serious events, requiring intensive supportive care and special care adapted to the poison. Therefore, laboratory diagnostics is needed in two different fields of activity. First, laboratory

*Corresponding author: Katharina M. Rentsch, Institute for Clinical Chemistry, University Hospital Zurich, Raemistrasse 100, 8091 Zuerich, Switzerland

Phone: +414425522 90, Fax: +414425545 90,

E-mail: rentsch@access.uzh.ch

Received January 8, 2010; accepted May 27, 2010;

previously published online July 27,2010 tests need to describe the pathophysiological state of the patient. At a minimum, these include coagulation tests, blood cell counts, blood gas tests, serum electrolytes, liver and kidney function tests, glucose, creatine kinase and osmolality. Except for osmolality, these are very common and fully automated tests which can usually be performed within a very short period of time. With these test results supportive treatment can be initiated.

As clinical symptoms of intoxications are usually not characteristic of a specific substance, in many cases subsequent toxicological analysis is needed unless anamnestic information provides enough evidence. There are very different indications for identifying the compounds involved in intoxications. Reasons include application of a specific therapy (e.g., antidote) for the poison, transfer of the patient to the intensive care unit or forensic reasons. Depending on the motivation for the investigation, the need for a short turn around time may be important. There are different strategies used and described for this purpose, depending on the methods available in the laboratory. For the most common drugs involved in intoxications, specific immunoassays for quantification in serum have long been available (e.g., acetaminophen, salicylates). Fully automated immunoassays are also available for the detection of the classic drugs of abuse. These immunoassays can be established in every clinical chemistry laboratory, but they can also be used in the emergency stations using point-of-care test devices. This allows these tests to be available in many clinical settings. Unfortunately, it is well known that these immunoassays for drugs of abuse have numerous technical problems, such as different cross-reactivities of the antibodies against drug metabolites, different compounds from the same drug class, or structurally similar substances from different drug classes. The highdose hook effect or the commonly applied cut-off concentrations that are well above the limits of detection of the respective tests are other factors which have to be considered when using these tests in clinical toxicology. In addition, new amphetamine-like designer drugs have entered the drugs of abuse scene [e.g., piperazines (1)], which cannot be detected by immunoassays. Thus, hyphenated chromatographic techniques using mass spectrometry as detection methods are widely used in specialised toxicological laboratories. Gas chromatography coupled to mass spectrometry (GC-MS) has been the gold standard since the early 1980s, and is still in common use today (2). In recent years, liquid chromatography coupled with mass spectrometry (LC-MS) has gained the interest of many researchers in clinical and forensic toxicology. This technique can be used in a more flexible way compared with GC-MS, and the turnaround time is usually much shorter (3-6). 
Colorimetric tests often can be performed very rapidly by adding a simple reagent to a patient sample, and are not burdened with high instrumental costs. The colour which is developed sometimes allows the identification of a single drug [e.g., phenothiazines with the Forrest test (7)]. Usually, these tests are less specific than chromatographic or immunological tests. Therefore, these tests are being replaced by these techniques in many laboratories which can afford the investment of immunoassay analyzers of chromatographic methods. However, they are routinely used for the identification of cyanide and iron in most laboratories.

Following identification of the substances involved in intoxication, often a quantitative or, at a minimum, a semiquantitative measurement is needed for adequate patient treatment. Quantifications of poisons should be performed in serum or plasma in order to enable correlation of the drug concentration with the degree of intoxication. Optimally, screening procedures enable estimation of the concentration of the poison. If this cannot be accomplished, a specific quantitative assay needs to be performed.

In the following sections, the most important and generally well-accepted parameters or procedures used in acute poisoning are reviewed, and their usefulness in different situations are discussed.

\section{Immunoassays}

Immunoassays are widely used in acute poisoning since they are widely available in many laboratories. The rating of these tests in acute clinical toxicology cannot be assessed in total. Immunoassay tests for therapeutic drugs or drugs of abuse are usually developed for a single matrix, e.g., urine or serum/plasma. One of the major reasons to do so is that the compound predominantly present in the particular matrix (drug and/or drug metabolite) which is detected by the assay is often not the same in blood and urine (8). The other important factor is the concentration range of the detected compound present in the respective matrix, which may vary widely. However, many clinical and forensic toxicological laboratories use the drugs of abuse tests developed for urine to analyze blood samples. If a laboratory decides to use an immunoassay for a matrix that it is not intended, a very careful evaluation of the assay needs to be performed including comparison of the results with a chromatographic method. Also, interpretation of the test results should be performed very carefully, taking into account the above mentioned caveats.

\section{Acetaminophen (paracetamol)}

If the intake of acetaminophen is suspected, quantitative determination of the drug in serum is highly recommended since initiation of antidotal therapy is dependent on the drug concentration. The use of a nomograph relating plasma drug concentration, time since ingestion and hepatotoxicity is helpful in the evaluation for the need for antidotal treatment (9). Blood collection should be performed at least $4 \mathrm{~h}$ after suspected intake of the drug. Acetaminophen is extensively metabolized, and only $1 \%-4 \%$ is excreted unchanged in urine following intake of therapeutic doses. In the case of an overdose, the amount that is excreted unchanged may increase to $10 \%-14 \%$ of the dose (10). The immunoassays that are used determine only acetaminophen itself, and do not show significant cross-reactivities with metabolites. There are enzymatic tests for quantification of acetaminophen in serum. Unfortunately, many of these tests are influenced by bilirubin and/or other compounds present in the serum of patients with liver injury (11). Polson et al. concluded that patients with acetaminophen intoxication usually do not have increased bilirubin concentrations when they are admitted to the hospital. In our experience, we occasionally see patients diagnosed with acute liver failure with pre-existing chronic liver impairment as a result of chronic infection with hepatitis viruses, and who have greatly increased bilirubin concentrations. Since acetaminophen is always a candidate for worsening of the disease, it is usually determined in these situations. This may result in incorrect interpretations if the assay being used is influenced by bilirubin or other compounds that are increased in chronic liver impairment (12).

\section{Digoxin}

In many countries, intoxication with digoxin has become very rare. However, the clinical symptoms may be very severe. Since there is a specific antibody available as an antidote, it is highly recommended that digoxin be measured whenever acute poisoning is suspected. Immunoassays used for quantification of this drug have been shown to interact with endogenous digoxin-like immunoreactive substances (DLIS). DLIS are increased in patients with volume expansion. This includes patients with uremia, essential hypertension, liver disease and preeclampsia. In addition, exogenous DLIS have been described with use of various Chinese medicines, spironolactone or canrenone (13). Digoxin is one of the compounds which usually cannot be determined using the general chromatographic unknown screening procedures, and therefore always needs to be analyzed specifically with immunoassays.

\section{Amanitin}

Amanitin is the toxin found in poisonous Amanita sp. mushrooms. A chromatographic method has been described to analyze this toxin (14), but the commercial immunoassay that is available is more widely used since it can be performed faster and does not require an LC-MS instrument. Due to the special chemical structure of amanitin, the immunoassay is very specific for this compound. This immunoassay is usually available in specialized toxicological laboratories in geographic areas where the mushroom grows. Because acute poisoning with Amanita sp. leads to very severe intoxication, antidotal therapy needs to be initiated before result from the laboratory are available. After ingestion of mushrooms containing amanitin, the clinical symptoms usually start within 16-24 h. The ability to detect amanitin in serum or plasma is about $30 \mathrm{~h}$ only, whereas it 
can be detected for up to 3 days in urine (15). Therefore, the assay should be performed using urine samples, but it is also acceptable to use serum or plasma.

\section{Drugs of abuse}

As mentioned above, immunoassays for the classic drugs of abuse are available in many hospital laboratories or emergency stations, have a short turn around time and are therefore, usually the first methods applied in toxicological screening procedures. The results need to be interpreted with utmost care as these tests have some major disadvantages when used in cases of acute poisoning despite their advantage of being widely available and having a short turn around time.

The primary disadvantage of the commonly used immunological tests for drugs of abuse in acute clinical toxicology is the application of an appropriate cut-off concentration for identifying the results as being "positive" or "negative". All tests performed with clinical chemistry instruments are calibrated and generate quantitative results. As the urine matrix can be very different between individuals, and the ratio between parent drug and metabolite(s) also can vary greatly, these results are usually considered to be semi-quantitative. Starting initially in the US workplace drug testing community, cut-off concentrations have been defined which are well above the limit of detection of the respective drug or drug class (16). Whenever the urine concentration of a drug is lower than the cut-off concentration, the result is considered to be "negative", despite the fact that in some cases the concentrations indicate the actual presence of this compound in the urine sample. This plays an important role in patients with polyuria, or in situations where the dosages needed to have a clinical effect diverge widely (e.g., benzodiazepines). I am not aware of any studies that describe the limit of detection of drugs of abuse tests from the different manufacturers. If a point-of-care device is used, the situation is even worse since the read-out for a positive result is only developed above the cut-off concentration. Furthermore, it should be mentioned that, especially in Europe, not all manufacturers use the same calibrator and often apply different cut-off concentrations. This can result in very different results if a urine sample is analyzed by different laboratories. In addition, a negative test result is usually considered to be correct, and if confirmation testing is performed, it is usually performed only for positive screening results.

The different cross-reactivities of antibodies with the different drugs or drug metabolites of a class of drugs can also lead to misinterpretation, especially in the case of negative results. Due to the different chemical structures of the drugs belonging to the benzodiazepines and amphetamines, screening for these two drug classes with immunoassays are usually the most troubled with this problem (17).

Several laboratories apply these drugs of abuse tests to serum or plasma samples (18). Due to the different concentrations of drugs in serum compared with urine, and the different patterns between drug and drug metabolites, this practice cannot be recommended if it is not intended by the manufacturer.

\section{Hyphenated chromatographic methods}

Hyphenated chromatographic methods, primarily GC-MS and LC-MS, are widely used in specialized toxicological laboratories for either dedicated quantification of one single compound [e.g., $\gamma$-hydroxybutyrate (GHB)], a class of compounds (e.g., glycols), a wide range of specific compounds (e.g., targeted screening), or in a more global way for general screening of unknown drugs.

Quantification of a single compound using hyphenated chromatographic methods is usually applied when the poison is known. However, several analytes require very specific procedure in order to be detected in patient samples (e.g., $\mathrm{GHB}$, colchicine). As these quantitative methods have similar properties to the classic therapeutic drug monitoring (TDM) methods using hyphenated chromatographic methods, they will not be discussed further in this review.

\section{General unknown screening}

General unknown screening (GUS) or systematic toxicological analysis (STA) is used to screen for unknown compounds in a patient's urine or serum. The technique used for this purpose needs to be able to separate and detect a wide variety of different compounds. The combination of highperformance liquid chromatography (HPLC) with diodearray detection (HPLC-DAD) or mass spectrometric detection (LC-MS or LC-MS/MS), or a GC with mass spectrometric detection (GC-MS) are all capable of performing a GUS procedure. To perform chromatographic separation of the analytes, GC requires hydrolysis of the very polar conjugate metabolites prior to analysis (2). HPLC methods usually can handle a wider range of polar substances if a gradient is applied to the mobile phase.

The identification of substances after chromatography depends on two different factors. First, the substance needs to have either UV absorption above $200 \mathrm{~nm}$ or must be ionisable before entering the MS. With respect to ionization, there are big differences between GC and HPLC methods, resulting in many more compounds which can be ionized after GC analyses as compared with HPLC. Second, the compound to be identified needs to be part of the library used for identification. There are commercial libraries available of DAD spectra containing approximately 3000 different spectra of potential compounds being found in cases of intoxication (19). For GC-MS, there are several commercial specialized libraries available for drugs, poisons, pesticides, pollutants and their metabolites containing $>7800$ spectra (20). Also, there are large libraries containing spectra from $>200,000$ chemical entities from very different fields (NIST07 Spectral Data, Wiley Registry of Mass Spectral Data). The commercially available libraries for HPLC-DAD and GC-MS can be applied for use on any instrument from any manufacturer, fulfilling the technical requirements for screening procedures. For LC-MS, at present there is no 
commercial library available and all research groups working on the establishment of LC-MS GUS methods need to develop their own library consisting of tandem mass spectra if using a triple-stage quadrupol or an ion trap instrument. Not only do the different ionization techniques [electrospray (ESI) vs. atmospheric pressure chemical ionization (APCI)] generate different mass spectra, but instruments from different manufacturers and from a single manufacturer with different construction of the mass analyzer may result in changes in fragmentation or in fragment intensity (21). If a time-of-flight instrument (TOF) is used, all masses reaching the detector can be seen in the chromatogram, independent of the presence of the corresponding mass in the library. This allows one to determine whether any additional substances are present in the chromatogram. TOF instruments did not allow generation of mass spectra (22), but new instruments combine a quadrupol with the time-of-flight technique (QTOF). Therefore, these instruments allow not only the detection of the exact mass of the parent ion, but Q-TOF instruments enable fragmentation of selected ions within the collision cell, followed by the measurement of the accurate mass spectrum of the fragments (23). If tandem mass spectrometry is used, the production of a MS/MS spectrum is usually only induced when the mass to charge ratio of the parent compound has been listed as a potential candidate compound. Depending on the concentration of a compound not envisaged for detection and identification, and the exact method applied in the mass spectrometer, it might be that this additional substance cannot be detected at all in the chromatogram. This makes the LC-MS/MS screening methods to targeted screening methods with a distinct number of compounds which can be identified (24). Dresen et al. have recently published an ESI-MS/MS library containing 1253 compounds, which could be applied not only on the type of instrument on which the spectra have been recorded, but also on two other instrument types from the same manufacturer using a linear ion trap for detection.

As the ionization process in LC-MS has limitations concerning the number of molecules which can be ionized at a given time, these methods are always at risk for ion suppression. This is true for both ionization techniques, ESI and APCI, but ESI is much more prone to ion suppression than APCI (25). If two substances are eluted concomitantly from the HPLC column, the substance that is ionized more easily will be more favorably ionized. The interfering compounds might derive from the matrix or from other exogenous compounds that may be present in higher concentrations. Especially in patients with intoxication, it is unknown which compounds are present and it could be fatal if one peak of a toxic compound is suppressed by the presence of another molecule. It is therefore mandatory for all LC-MS GUS procedures to carefully test for ion suppression using real patient samples (26).

If general screening for unknown compounds is performed, the information about the detected compounds is very important. Not all the methods described above allow detection of every substance potentially involved in intoxications. Organic solvents (e.g., ethanol, methanol or ethylen- glycol) and metals (e.g., lithium) cannot be detected by any of the above mentioned screening methods. The identification of other substances is highly dependent on the extraction method used and the analytical technique applied, as well as the library used for the identification of the unknown compound. Therefore, it is mandatory that the specialized toxicological laboratory has a GUS strategy established which consists of immunoassays, enzymatic tests and different chromatographic protocols to allow GUS in a potentially poisoned patient. In rare situations, atomic absorption spectrometry (AAS) or inductively coupled plasma-mass spectrometry (ICP-MS) should be available for the identification of metals. In Figure 1, a graph is shown representing the GUS strategy in our laboratory. A thorough discussion of each case between the toxicologist performing the analyses and the physician treating the patient is mandatory.

\section{Targeted screening}

Identification and confirmation of positive drugs of abuse screening tests in urine have long been performed using GCMS. In recent years, many laboratories have changed their methods for drugs of abuse confirmation to LC-MS/MS, and also have developed analytical methods for screening tests for drug classes, such as antidiabetic drugs, diuretics or laxative agents (27). Usually these assays use selected reaction monitoring (SRM) observing one or more transitions. It should be considered that the different compounds may have one common transition resulting in interference, as recently shown by Sauvage et al. (28). These targeted screening procedures are usually applied when there is some evidence that the respective drug class is involved in cases of intoxication.

\section{Quantification of the poison}

As the concentration of the poison often helps in deciding on treatment of the patient an estimation of the identified compound(s) is often needed. If urine is used as sample material for screening, estimation of the concentration of the poison in urine gives only a rough estimate about the ingested amount of the drug. If serum or plasma is used for screening purposes, direct quantification can be performed (29). The analytical method for quantification needs to be fully validated in order to ensure correct results. As it is not possible to have fully validated quantitative methods for all possible poisons available, in many cases a semi-quantitative estimation of the concentration based, for example, on a onepoint calibration or on the standard addition method can be helpful.

As the absorption of a given compound at a distinct wavelength in UV spectrophotometry is dependent solely on its concentration, GUS procedures using HPLC-DAD detection enable a semi-quantitative estimation of the drug concentration by dividing the peak area in the sample chromatogram by the specific peak area, measured in $1 \mu \mathrm{g} / \mathrm{mL}$ of the analyte under the same conditions. The specific conditions and recovery of the sample preparation need to be considered using the corresponding correction factors. 


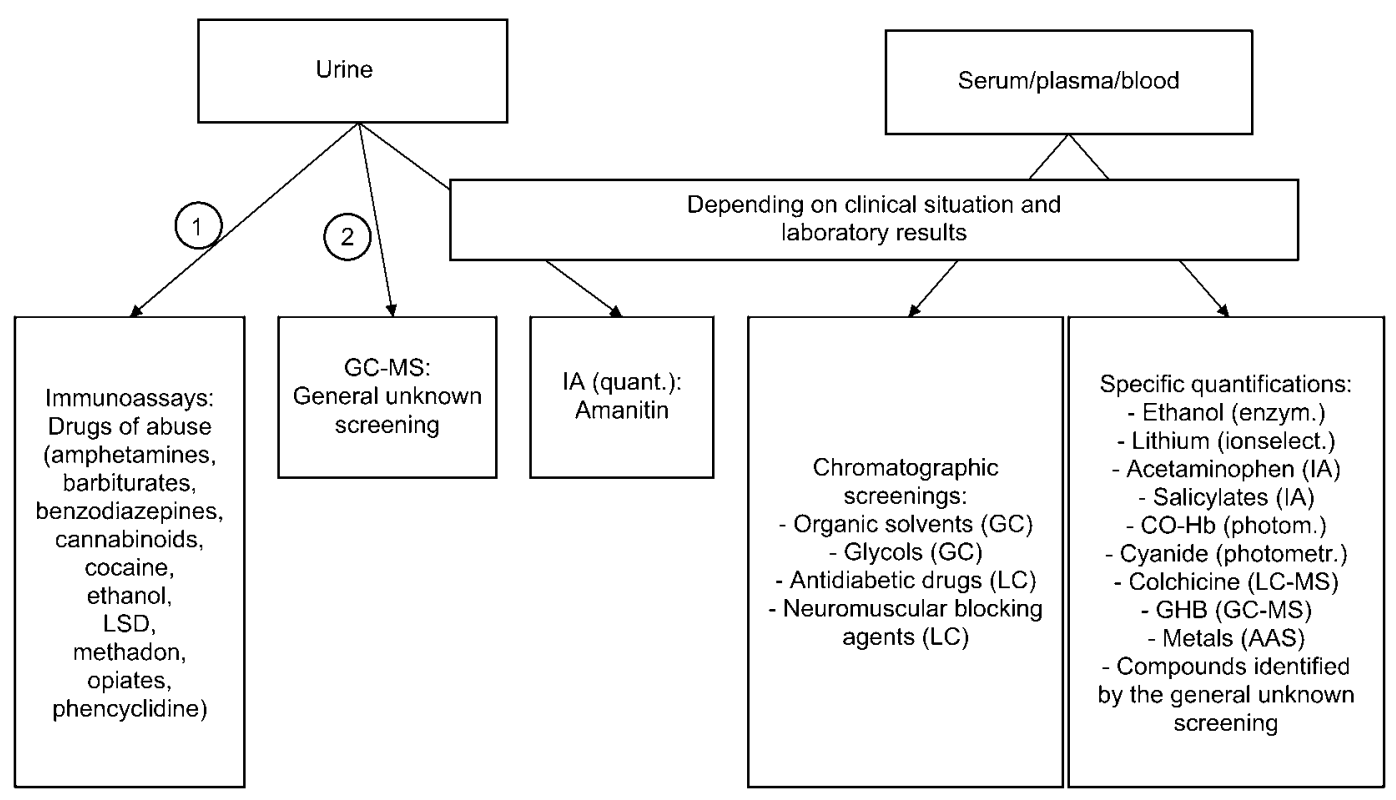

Figure 1 Example of an analytical strategy for the elucidation of an acute poisoning.

Enzym, enzymatic reaction; GC-MS, gas chromatography mass spectrometry; LC-MS, liquid chromatography mass spectrometry; IA, immunoassay; ionselect., ion selective electrode; $\mathrm{CO}-\mathrm{Hb}$, CO-hemoglobin; photometr., photometry; quant., quantitative; AAS, atomic absorption spectrometry.

Whereas many compounds involved in intoxication can be obtained commercially, others are difficult to acquire. In these situations, the companies producing the compounds can sometimes provide the laboratory with these standards or commercial drug formulations (e.g., tablets) that can be extracted. In the latter case, the concentrations determined using these calibrators should only be considered to be semiquantitative as the purity of the standard is not known.

\section{Identification of metals}

\section{Lithium}

Lithium is a toxic metal and the severity of intoxication corresponds to its concentrations in serum. Quantification of lithium with ion selective electrodes or flame emission photometry should be performed whenever the intake of this drug is considered.

\section{Iron}

Poisoning with iron is rare and almost always involves children who discover their mother's pink iron supplements, and then consume them like candy. The quantification of iron is usually performed with use of colorimetric tests which are available on clinical chemistry instruments.

\section{Arsenic, mercury, thallium}

Poisonings with arsenic are very rare in Europe, but are seen more often in developing countries. There are still arsenic containing herbicides and pesticides available, which then may be used in suicide or homicide. In addition, acute intox- ication with mercury is very rare, and is due to inhalation of vapor or dust containing mercury. Thallium is easily absorbed from the gastrointestinal tract and is distributed throughout the body. In the blood compartment, thallium can be found in erythrocytes. All of these metals are usually analyzed using AAS or ICP-MS. ICP-MS allows for the simultaneous measurement of multiple metal ions, which makes the technique suitable for screening purposes. When AAS or ICP-MS instruments are not available, cooperation with an institution that regularly uses these techniques should be established.

\section{Identification of solvents and chemicals}

\section{Organic solvents}

Besides the popular use of ethanol, the use of organic solvents in intoxication has several reasons. Sometimes, a solvent may be drunk mistakenly if it was stored in a beverage bottle, or if self-produced liquors erroneously contain methanol. Other times, organic solvents may be used as poisons in suicide attempts. More recently, the abuse of solvents by sniffing has become more popular, and may lead to intoxication. During the first phase after intake of solvents, these compounds add to the osmolality, and their presence and the amount taken can be estimated by calculating the osmolal gap. The organic solvents most often detected in cases of intoxication (methanol and ethylene glycol) are metabolized to organic acids which do not contribute to the osmolality, but do result in an increased anion gap.

The organic solvents can be determined by GC coupled to different detectors (e.g., flame ionization or mass spectrom- 
etry) in specialized toxicological laboratories. The organic acid metabolites can, for example, be quantified by ion chromatography.

\section{$\gamma$-Hydroxybutyrate (GHB)}

GHB is often used as a drug of abuse or as a date-rape drug. It has a very short elimination half-life, which allows detection in serum or plasma for about $6 \mathrm{~h}$, and in urine for about $12 \mathrm{~h}$ only. GHB usually is determined with a specific GCMS method after derivatization, such as silylation (30).

\section{Cyanide}

Cyanide is a very rare poison usually involved in cases of suicide or homicide. Colorimetric tests enable fast and reliable quantification of cyanide in serum and urine. Colorimetric, fluorimetric and chromatographic methods have been described for the analyses of cyanide in biological fluids. A simple and fast colorimetric method for the quantification of cyanide in blood uses the reaction of cyanide with 1,2-dinitrobenzene and 4-nitrobenzaldehyde in 2-methoxyethanol to produce 2-nitrophenylhydroxylamine (31).

\section{Conclusions}

Laboratory diagnostics is essential in acute poisoning. Early following admission to the hospital, general clinical chemistry and hematology tests should be performed to determine the pathophysiological state of the patient. In addition, there are many situations where the identification and sometimes the quantification of the compound(s) involved are useful and necessary. There are some situations where an antidote can be started, such as intoxication with acetaminophen, digoxin or amanitin. At other times, GUS procedures are necessary when the anamnestic information does not provide enough evidence that all compounds taken by the patient are known. Independent of the analytical technique used (HPLCDAD, GC-MS and LC-MS), the limitations concerning detection of the different compounds, the number of analytes present in the library and the knowledge about the limitation of the technique must be considered in the interpretation of the results of a screening method. Usually, different methods need to be used concomitantly in order to obtain reliable results. Often, it is necessary not only to identify the poison, but also to quantify its concentration in serum or plasma in order to assure adequate treatment of the patient.

\section{Conflict of interest statement}

Author's conflict of interest disclosure: The author stated that there are no conflicts of interest regarding the publication of this article.

Research funding: None declared.

Employment or leadership: None declared.

Honorarium: None declared.

\section{References}

1. Maurer HH, Kraemer T, Springer D, Staack RF. Chemistry, pharmacology, toxicology, and hepatic metabolism of designer drugs of the amphetamine (ecstasy), piperazine, and pyrrolidinophenone types: a synopsis. Ther Drug Monit 2004;26:12731.

2. Maurer HH. Systematic toxicological analysis of drugs and their metabolites by gas chromatography-mass spectrometry. J Chromatogr 1992;580:3-41.

3. Sauvage FL, Sain-Marcoux F, Duretz B, Deporte D, Lachatre G, Marquet P. Screening of drugs and toxic compounds with liquid chromatography-linear ion trap tandem mass spectrometry. Clin Chem 2006;52:1735-42.

4. Lee HK, Ho CS, Io YP, Lai PS, Shek CC, Lo AC, et al. Development of a broad toxicological screening technique for urine using ultra-performance liquid chromatography and time-offlight mass spectrometry. Anal Chim Acta 2009;649:80-90.

5. Liu HC, Liu RH, Lin DL, Ho HO. Rapid screening and confirmation of drugs and toxic compounds in biological specimens using liquid chromatography/ion trap tandem mass spectrometry and automated library search. Rapid Commun Mass Spectrom 2010;24:75-84.

6. Dresen S, Ferreiros N, Gnann H, Zimmermann R, Weinmann W. Detection and identification of 700 drugs by multi-target screening with a 3200 Q TRAP LC-MS/MS system and library searching. Anal Bioanal Chem 2010;396:2425-34.

7. Forrest IS, Forrest FM. Urine colour test for the detection of phenothiazine compounds. Clin Chem 1960;6:11-5.

8. Kraemer T, Paul LD. Bioanalytical procedures for determina-

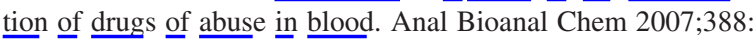
1415-35.

9. Rumack BH, Peterson RG. Acetaminophen overdose: incidence, diagnosis, and management in 416 patients. Pediatrics 1978;62:898-903.

10. Slattery JT, Levy G. Acetaminophen kinetics in acutely poisoned patients. Clin Pharm Ther 1979;25:185-95.

11. Polson J, Wians FH Jr, Orsulak P, Fuller D, Murray NG, Koff $\mathrm{JM}$, et al. False positive acetaminophen concentrations in patients with liver injury. Clin Chim Acta 2008;391:24-30.

12. Bertholf RL, Johannsen LM, Bazooband A, Mansouri V. Falsepositive acetaminophen results in a hyperbilirubinemic patient. Clin Chem 2003;49:695-8.

13. Dasgupta A. Endogenous and exogenous digoxin-like immunoreactive substances: impact on therapeutic drug monitoring of digoxin. Am J Clin Pathol 2002;118:132-40.

14. Maurer HH, Schmitt CJ, Weber AA, Kraemer T. Validated electrospray liquid chromatographic-mass spectrometric assay for the determination of the mushroom toxins alpha- and beta-amanitin in urine after immunoaffinity extraction. J Chromatogr B Biomed Sci Appl 2000;748:125-35.

15. Vesconi S, Langer M, Iapichino G, Costantino D, Busi C, Fiume L. Therapy of cytotoxic mushroom intoxication. Crit Care Med 1985;13:402-6.

16. Killander J, de la Torre R, Segura J, de Zeeuw R, Williams J. Recommendations for the reliable detection of illicit drugs in urine, with special attention to the workplace, in the European Union (December 1996). The Toxicology Experts' Working Group. Scand J Clin Lab Invest 1997;57:97-103.

17. Hsu J, Liu C, Liu CP, Tsay WI, Li JH, Lin DL, et al. Performance characteristics of selected immunoassays for preliminary test of 3,4-methylenedioxymethamphetamine, methampheta- 
mine, and related drugs in urine specimens. J Anal Toxicol 2003;27:471-8.

18. Schutz H, Auch J, Erdmann F, Weiler G, Verhoff MA. Reliability of the cloned-enzyme donor immunoassay (CEDIA) for cocaine in human serum in the range between the detection limit and the cut-off. Arzneimittelforschung 2006;56:414-20.

19. Pragst F, Herzler M, Herre S, Erxleben B-T, Rothe M. UV spectra of toxic compound: database of photodiode array UV spectra of illegal and theraputic drugs, pesticides, ecotoxic substances and other poisons, 1st ed. Berlin: Verlag Toxicological Chemistry, 2008:408 pp.

20. Maurer HH, Pfleger K, Weber AA. Mass spectral and GC data of drugs, poisons, pesticides, pollutants and their metabolites, 3rd ed. Weinheim: Wiley-VCH, 2007:1432 pp.

21. Jansen R, Lachatre G, Marquet P. LC-MS/MS systematic toxicological analysis: comparison of MS/MS spectra obtained with different instruments and settings. Clin Biochem 2005; 38:362-72.

22. Ojanpera S, Pelander A, Pelzing M, Krebs I, Vuori E, Ojanpera I. Isotopic pattern and accurate mass determination in urine drug screening by liquid chromatography/time-of-flight mass spectrometry. Rapid Commun Mass Spectrom 2006;20:1161-7.

23. Decaestecker TN, Vande Casteele SR, Wallemacq PE, van Peteghem $\mathrm{CH}$, Defore DL, van Bocxlaer JF. Informationdependent acquisition-mediated LC-MS/MS screening procedure with semiquantitative potential. Anal Chem 2004;76: 6365-73.

24. Dresen S, Gergov M, Politi L, Halter C, Weinmann W. ESIMS/MS library of 1253 compounds for application in forensic and clinical toxicology. Anal Bioanal Chem 2009;395:2521-6.
25. Van Eeckhaut A, Lanckmans K, Sarre S, Smolders I, Michotte Y. Validation of bioanalytical LC-MS/MS assays: evaluation of matrix effects. J Chromatogr B Analyt Technol Biomed Life Sci 2009;877:2198-207.

26. Chambers E, Wagrowski-Diehl DM, Lu Z, Mazzeo JR. Systematic and comprehensive strategy for reducing matrix effects in LC/MS/MS analyses. J Chromatogr B Analyt Technol Biomed Life Sci 2007;852:22-34.

27. Maurer HH, Kratzsch C, Kraemer T, Peters FT, Weber AA. Screening, library-assisted identification and validated quantification of oral antidiabetics of the sulfonylurea-type in plasma by atmospheric pressure chemical ionization liquid chromatography-mass spectrometry. J Chromatogr B Analyt Technol Biomed Life Sci 2002;773:63-73.

28. Sauvage FL, Gaulier JM, Lachatre G, Marquet P. Pitfalls and prevention strategies for liquid chromatography-tandem mass spectrometry in the selected reaction-monitoring mode for drug analysis. Clin Chem 2008;54:1519-27.

29. Kratzsch C, Weber AA, Peter FT, Kraemer T, Maurer HH. Screening, library-assisted identification and validated quantification of fifteen neuroleptics and three of their metabolites in plasma by liquid chromatography/mass spectrometry with atmospheric pressure chemical ionization. J Mass Spectrom 2003;38:283-95.

30. Louagie HK, Verstraete AG, De Soete CJ, Baetens D, Calle PA. A sudden awakening from a near coma after combined intake of gamma-hydroxybutyric acid (GHB) and ethanol. Clin Toxicol 1997;35:591-4.

31. Vesey CJ, McAllister H, Langford RM. A simple, rapid and sensitive semimicro method for the measurement of cyanide in blood. Ann Clin Biochem 1999;36:755-8. 